\title{
Mixed-modality psychophysical scaling: Double cross-modality matching for "difficult" continua
}

\author{
LAWRENCE M. WARD \\ University of British Columbia, Vancouver, British Columbia, Canada
}

\begin{abstract}
In mixed-modality psychophysical scaling, stimuli from different modalities are presented alternately for judgment on the same scale. The usual purpose is to produce cross-modality matching functions without actually doing cross-modality matches. This paper reports the results of two experiments that extend the method to situations in which the responses, themselves crossmodality matches on an easy-to-control continuum (duration), are used to derive matching functions for two difficult-to-control continua (here loudness and brightness). Derived cross-modality matching functions are highly similar to those obtained from magnitude estimation or category judgment responses. First- and second-order sequential dependencies also closely resemble those found in data from the methods that employ numerical response scales, with one exception. For the first time in these studies of mixed-modality scaling, current responses sometimes were found to be weakly contrasted to the values of previous stimuli of different modality from the current stimulus. The various sequential dependencies found may arise from different levels of processing, with intramodal response-stimulus contrast arising from sensory differentiation, inter- and intramodality response-response assimilation from perceptual categorization processes, and intermodality response-stimulus contrast from cognitive expectancies.
\end{abstract}

In mixed-modality psychophysical scaling, subjects make judgments of two or more different sensory continua on the same scale (Ward, 1982). The first such method, magnitude matching, was introduced by J. C. Stevens and Marks (1980) to allow cross-modality matching functions to be derived for the judged continua without the necessity of actually controlling intensities on one of the continua. Subjects simply made magnitude estimations of stimuli from both continua, and the cross-modality matching function was generated by selecting intensities on the two continua that had received judgments of the same magnitude. This method sidesteps the problems inherent in having subjects adjust the intensity of one continuum until it matches that of another. For example, continuous adjustments are impractical for some modalities, such as smell and taste, and the method is too timeconsuming for continua characterized by rapid adaptation, such as brightness, thermal sensation, and again taste and smell, in which judgments must be separated by long rest periods. Furthermore, J. C. Stevens and Marks (1980) demonstrated that magnitude matching could be expected to be superior to ordinary magnitude estimation in diagnosing sensory pathology, and that it was capable of differentiating between types of deafness with the same precision as is ordinary cross-modality matching.

This research was supported by Grant A9958 from the Natural Sciences and Engineering Research Council of Canada. I thank Barry Chew, Elsie Cheung, and Odie Geiger for assistance in collecting and analyzing the data. Reprint requests should be sent to Lawrence $\mathbf{M}$. Ward, Department of Psychology, University of British Columbia, 2136 West Mall, University Campus, Vancouver, B.C., Canada V6T 1 Y7.
Other variants of mixed-modality scaling may prove to be equally useful. Ward $(1982,1985)$ has demonstrated that category matching, in which subjects make category judgments of two or more continua on the same category scale, produces cross-modality matching functions that are statistically indistinguishable from those produced by magnitude matching. Also, category judgments tend to vary less between subjects, so that they may be even more powerful in detecting sensory deficits. Neither method is very sensitive to the sequence in which the different continua are presented, and both yield data that are useful in differentiating between theories of psychophysical judgment. Interestingly, although both methods are subject to the usual biases of judgment, such as first- and secondorder sequential dependencies, such biases have little effect on the slopes of cross-modality matching functions.

The purpose of the present paper is to present results that extend even further the usefulness of mixed-modality psychophysical scaling. One of the advantages of crossmodality matching, that of the lack of a requirement for a verbal or numerical response, is missing in magnitude and category matching. It seems important to ask whether there is a way of generating cross-modality matching functions that will be as convenient as magnitude or category matching and yet will not require verbal or numerical responses. One natural solution is to have subjects match each of two or more "difficult" continua to an "easy" continuum. This allows the calculation of derived crossmodality matching functions for the "difficult" continua while avoiding a requirement for verbal or numerical responses and calculations on them, thus preserving the 
advantages of direct cross-modality matching. Similar procedures have been suggested before (e.g., Krantz, 1972; S. S. Stevens, 1975), but only for one continuum at a time and without generating derived cross-modality matching functions from the direct matching functions. In this paper, I report results of two experiments that not only establish the viability of this approach, but also provide an empirical puzzle for theorists of psychophysical judgment.

In this approach, choice of a response continuum is crucial. There are several continua that might be useful, including force of handgrip (e.g., S. S. Stevens, 1975), line length (Daning, 1983; Teghtsoonian \& Teghtsoonian, 1983), area (Baird, Green, \& Luce, 1980), finger span (Daning, 1983), lifted weight (Daning, 1983), and duration (Ward, 1975, 1979). Duration, in particular, seems to have many properties that make it a convenient response continuum. First, magnitude estimations are roughly linear with duration (S. S. Stevens, 1975; Ward, 1975), so that duration can act as a sort of analog substitute for numerical magnitude. It is easy for the subject to vary perceived duration continuously, for example by pressing a button twice, with the interval between presses forming the desired time-interval response. There is no need for multiple presentations of each stimulus, or multiple opportunities to adjust each response, since subjects quickly learn to produce an acceptable response in a single try. There is no sensory adaptation per se for duration, so the only adaptation effects of concern are those on the continua to be judged. Finally, subjects are free to choose the range of responses they wish to use, subject to the desire to finish the experiment in a reasonable time, since there is no necessary upper limit for duration, such as discomfort threshold in other modalities. Because of these properties, duration between two keypresses was chosen as the response continuum for the experiments reported in this paper.

These experiments address several other issues as well. Ward $(1982,1985)$ reported that in mixed-modality scaling situations in which responses were magnitude estimates or category judgments, current responses were assimilated to previous responses up to a lag of 4 regardless of the modality of the stimuli responded to, whereas, with only minor exceptions, current responses to stimuli of a given modality were contrasted to previous stimulus intensities only of the same modality and again up to a lag of 4. It is known that similar sequential dependencies characterize cross-modality matches (Baird et al., 1980; Ward, 1975, 1979), but it is not known whether the predominantly intramodality nature of response-stimulus contrast effects will be the case for cross-modality matches. A few differences have been noted between cross-modality matches and other forms of judgment (e.g., in second-order dependencies; see Baird et al., 1980), and it is of some importance to several theoretical approaches that the exact relationship between the various scaling methods be explored. The data from the experiments reported here were thus also analyzed for first- and second-order sequential dependencies, and the results of these analyses were compared with those for magnitude and category matching.

In order to accomplish the above-described goals, two experiments were run in which duration between keypresses was matched to brightness of lights and loudness of sounds. Experiment 1 was run using the standard mixed-modality method in which stimuli from different modalities are presented in strict alternation for judgment on the same scale. Thus, in Experiment 1, a segment of a typical sequence of stimuli and responses looked like

$$
\ldots S_{n-3} R_{n-3} L_{n-2} R_{n-2} S_{n-1} R_{n-1} L_{n} R_{n} \ldots \text {, }
$$

where $L_{n-k}$ represents a light stimulus, $S_{n-k}$ a sound stimulus, and $R_{n-k}$ a response, on Trial $n-k$. Ward (1985) pointed out that this sequence does not allow a complete analysis of sequential dependencies as a function of lag, and so Experiment 2 was run using a different stimulusresponse sequence. A typical segment of this "alternatingby-pairs" sequence would look like

$$
\begin{aligned}
\ldots S_{n-7} R_{n-7} S_{n-6} R_{n-6} L_{n-5} R_{n-5} L_{n-4} R_{n-4} \\
\\
S_{n-3} R_{n-3} S_{n-2} R_{n-2} L_{n-1} R_{n-1} L_{n} R_{n} \ldots,
\end{aligned}
$$

where $S_{n-k}, L_{n-k}$, and $R_{n-k}$ have the same meanings as in the standard sequence (see Ward, 1985, for a detailed description). Here subjects make two successive judgments of stimuli from one modality followed by two successive judgments of stimuli from the other, and so forth. Experiment 2 also provides a replication of several important aspects of Experiment 1, and, as will be seen, this turns out to have been useful.

\section{METHOD}

\section{Experiment 1: Standard Sequence}

Subjects. Eight subjects with normal or corrected-to-normal vision and no known hearing defects participated for pay. Four were females and 4 were males.

Apparatus. A standard sequence of sounds and lights was presented for judgment. The apparatus was identical to that of Ward (1982). Briefly, for the light stimuli a $1.5^{\circ}$ dot of approximately uniform luminance was produced at about eye level for the subject, who sat in a Model 250 IAC chamber. The 10 light intensities used were $167.6,84.00,42.10,21.10,10.58,5.300,2.656$, $1.331,0.667$, and $0.334 \mathrm{~cd} / \mathrm{m}^{2}$. Adjacent intensities were thus separated by $0.3 \log$ units $(3 \mathrm{~dB})$. For the sound stimuli, a $1000-\mathrm{Hz}$ tone was delivered diotically through stereo headphones at one of the following sound pressure amplitudes: $11.25,6.324,3.556$, $2.000,1.125,0.632,0.356,0.200,0.113$, or $0.063 \mathrm{dynes} / \mathrm{cm}^{2}$. Adjacent amplitudes were thus separated by $0.25 \log$ unit $(5 \mathrm{~dB})$. These ranges of light and sound are roughly equivalent in terms of proportions of the dynamic range and are in the range of those used by J. C. Stevens and Marks (1980) in their study of magnitude matching. Each stimulus was presented for $1 \mathrm{sec}$. Responses were indicated by two successive presses of a key on a standard computer keyboard illuminated by a dim red light. A PDP-11/34A computer system controlled stimulus presentation and recorded responses.

Procedure. The procedure was identical to that of Ward (1982) except for the type of response made by the subjects. Briefly, the subjects made a total of 1,200 judgments (600 each of light and sound, about 60 per intensity level) in four sessions of 300 judg- 
ments each, after 200 practice judgments. Stimulus intensity was chosen at random on each trial. The subjects were dark-adapted for about $2 \mathrm{~min}$ before the start of each session. However, since presentations of light stimuli were separated by only about 10 to $14 \mathrm{sec}$, complete dark adaptation probably did not occur following presentation of the most intense light stimuli.

The subjects received instructions identical to those of Ward (1982), which were adapted from those of J. C. Stevens and Marks (1980), except that they were told to make their responses by generating a duration between two keypresses. The instructions emphasized that subjects were to attempt an "absolute" match of the duration generated to the sensation intensity given rise to by each stimulus. The subjects had no trouble adopting an efficient strategy for generating the required durations. Durations used as responses ranged between about $250 \mathrm{msec}$ (approaching the shortest duration that could be generated by two successive keypresses) and $12 \mathrm{sec}$, with most subjects selecting a range of about $1.5 \log _{10}$ units within this larger range.

\section{Experiment 2: Alternating-by-Pairs Sequence}

Subjects. Eight different subjects with normal or corrected-tonormal vision and no known hearing defects participated for pay. Five were males and 3 were females.

Apparatus. All stimuli and equipment were identical to those used in Experiment 1.

Procedure. The procedure was identical to that of Experiment 1 except that stimuli were presented in the alternating-by-pairs sequence. Instructions were identical to those of Experiment 1 except that subjects were told of the alternating-by-pairs sequence instead of the standard sequence.

\section{RESULTS}

\section{Derived Cross-Modality Matching Functions}

Figure 1A shows the cross-modality matching function for light and sound calculated from the data of Experiment 1 using the procedure of J. C. Stevens and Marks (1980). Individual functions were highly similar to this group average function. All matching functions were reasonably well fitted by simple power functions (straight lines in decibel coordinates). The average slope (exponent) of the individual matching functions was 1.79 , which is close to the slope (exponent) of the group function in Figure 1A (1.70) and to the corresponding slopes (exponents) obtained by Ward $(1982,1985)$ for magnitude and category matching. (Note that the slopes reported here are the geometric means of the sound-on-light slopes and the reciprocals of the light-on-sound slopes as reported by J. C. Stevens and Marks (1980, Appendix 2). Figure 1 displays only the sound-on-light regression equation.) Using direct cross-modality matches of each "difficult" continuum to a third "easy" continuum has produced derived matching functions that are highly similar to those produced by magnitude and category matching. Furthermore, these matching functions were produced conveniently and quickly, but without the requirement of verbal or numerical responses. Double cross-modality matching on "easy" continua is a useful method for producing matching functions for "difficult" continua, especially when verbal or numerical responses are undesirable.

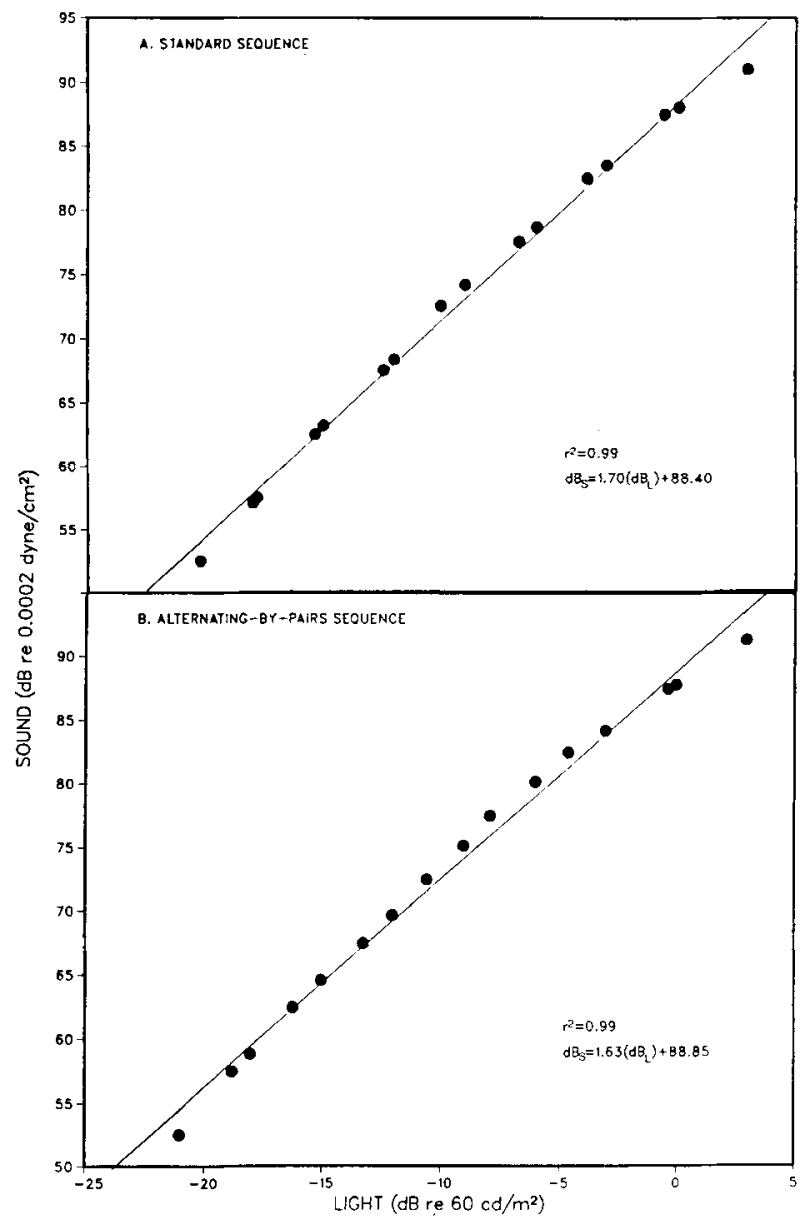

Figure 1. Cross-modality matching functions generated from the standard sequence $(A)$ and alternating-by-pairs sequence $(B)$ data. The straight lines through the points are the lines whose equations are printed on the figure.

Figure 1B shows the group average indirect crossmodality matching function for light and sound calculated from the data of Experiment 2, which employed the alternating-by-pairs stimulus-response sequence. Again, individual subjects' matching functions were highly similar to the group function displayed in Figure 1B. The average slope (exponent) of the individual matching functions was 1.65, again close to that of the group function (1.63) and to those obtained by Ward $(1982,1985)$.

Table 1 shows the average of the slopes (exponents) of individuals' derived matching functions for light and sound from all of the mixed-modality experiments reported from my laboratory so far, including those of the present paper and those of Ward $(1982,1985)$. Analysis of variance of the individual exponents from all six of these experiments, each employing a different group of subjects, reveals that neither response method $[F(2,42)$ $=1.46]$ nor type of sequence $[F(1,42)=0.30]$ has a reliable effect on the exponent of the matching function, and that the two variables do not interact $[F(2,42)=$ 
Table 1

Averages (Standard Deviations) of Exponents of Individuals' Indirect Matching Functions

\begin{tabular}{lcc}
\hline \multicolumn{1}{c}{ Method } & $\begin{array}{c}\text { Standard } \\
\text { Sequence }\end{array}$ & $\begin{array}{c}\text { Alternating-by-Pairs } \\
\text { Sequence }\end{array}$ \\
\hline Magnitude Matching & $1.60(0.38)^{*}$ & $1.74(0.31) \dagger$ \\
Category Matching & $1.52(0.36)^{*}$ & $1.57(0.20) \dagger$ \\
Cross-Modality Matching & $1.79(0.38) \ddagger$ & $1.65(0.15) \ddagger$ \\
\hline
\end{tabular}

*Ward (1982) †Ward (1985) ‡this study

$0.84]$. This conclusion is not the result of large variances, inasmuch as the standard deviations displayed in Table 1 are quite modest (the mean squared error within subjects for the analysis of variance was 0.096). These derived matching functions seem quite resistant to biases peculiar to the response continuum employed.

\section{Microanalysis of Duration Responses}

Multiple regression analyses. A useful summary of dependencies of scaling responses on previous stimuli and responses is provided by estimation of $\gamma, \alpha_{i}$, and $\beta_{k}$ in

$$
\begin{aligned}
\log R_{n}= & \gamma \log I_{n}+\sum_{i=1}^{M} \alpha_{i} \log I_{n-i} \\
& +\sum_{k=1}^{N} \beta_{k} \log R_{n-k}+\delta+\epsilon
\end{aligned}
$$

(see Jesteadt, Luce, \& Green, 1977; Ward, 1979, 1982, 1985). These constants were estimated for the present data by hierarchical linear regression, in which the independent variables were entered into the regression in the order in which they occurred in the sequence of stimuli and responses. For the coefficients reported here, $M$ and $N$ were set equal to 4 because individual subjects' coefficients were seldom reliable for higher lags. Because of the difficulty of interpreting the reliability of individual subjects' coefficients, conclusions are based mostly on $t$ tests of average coefficients over the groups of subjects, and on the increase in $R^{2}$ when the various previous stimuli and responses were added hierarchically to the regression.

Tables 2 and 3 summarize the results of these analyses for the standard sequence data of Experiment 1. In all of these analyses, responses to sounds were analyzed separately from responses to lights. For example, Row $S$ in Table 2 contains average regression coefficients for responses when $I_{n}$ was a sound (e.g., $S_{n}$ ). For such trials, $R_{n-1}$ was always a duration match to a light, $L_{n-1}, R_{n-2}$ was always a duration match to a sound, $S_{n-2}$, and so forth for the remainder of the coefficients. The coefficients in Row $L$ have the complementary interpretation.

The estimate of $\gamma$ in Row $S$ of Table 2 represents the standard power function exponent for sound, which, in this case, is somewhat smaller than the 0.67 usually taken to be typical for sound pressure. Since duration and number are approximately linearly related, we would expect roughly the same exponent for duration matching and magnitude estimation, yet the exponent is closer to that found for category judgment (see, e.g., Marks, 1974; Ward, 1972). The estimates of $\beta_{1}, \beta_{2}, \beta_{3}$, and $\beta_{4}$ are all positive and highly reliably different from zero, indicating that the current response is assimilated to previous responses up to a lag of 4, regardless of the modality of the stimulus to which the previous response was made. This result is consistent with those of previous studies of magnitude and category matching (Ward, 1982, 1985) and with those of other studies of cross-modality matching (e.g., Baird et al., 1980; Ward, 1975). Ward (1982) did not report coefficients for lags greater than 2 , but an examination of those data for lags up to 4 reveals the same pattern. Although the regression coefficients are reliably different from zero up to a lag of 4 , Table 3 shows that only $R_{n-1}$ adds a large amount to $R^{2}(0.052$ in these data). This is also true for category and magnitude matching, although $R_{n-1}$ is less influential in those data, seldom adding more than 0.02 to $R^{2}$. Clearly, $R_{n-1}$, regardless of the modality of its stimulus, is the most influential of the previous responses. Furthermore, it is about twice as influential for cross-modality matching with duration as the response continuum, possibly because durations are not easy to produce precisely (see Ward, 1975), allowing other sources of variance to be more important. Consistent with this, the $R^{2}$ increase for sound $I_{n}$ in this study $(0.588)$ is lower than those found in any of the previous mixed-modality studies $(0.684$ and 0.801 for magnitude and category matching, respectively, from Ward, 1982 , and $0.741,0.692$ for magnitude matching and 0.802 , 0.772 for category matching from Ward, 1985).

Now look at the $\alpha_{i}$ coefficients of Row $S$ in Table 2 . As for previous studies of mixed-modality scaling, the estimates of $\alpha_{2}$ and $\alpha_{4}$ are negative and reliably different from zero, indicating that the current response was contrasted with earlier stimuli of the same modality. Moreover, these stimuli increase $R^{2}$ significantly when added to the regression, almost 0.03 for $I_{n-2}$ and almost 0.01 for $I_{n-4}$. There is also a contrastive effect of $I_{n-1}$ which adds over 0.01 to $R^{2}$, but the regression coefficient is much smaller than it is for the intramodality contrast effects. This is the first time an intermodality contrast effect has been observed that adds so much to $R^{2}$. Ward (1985) found two intermodality contrast effects of similar coefficient magnitude, but they added only 0.000 and 0.002 to $R^{2}$ and were clearly minor exceptions to the rule of intramodality contrast. Because an effect of the same relative importance also occurs for light stimuli (see Row $L$ of Ta-

Table 2

Average Regression Coefficients from Equation 1 for Standard Sequence Data

\begin{tabular}{lccccccccc}
\hline & $\gamma$ & $\beta_{1}$ & $\alpha_{1}$ & $\beta_{2}$ & $\alpha_{2}$ & $\beta_{3}$ & $\alpha_{3}$ & $\beta_{4}$ & $\alpha_{4}$ \\
\hline$S$ & $.326^{*}$ & $.225^{*}$ & $-.026 \dagger$ & $.176^{*}$ & $-.080 \dagger$ & $.080^{*}$ & -.017 & $.117^{*}$ & $-.055^{*}$ \\
$L$ & $.278^{*}$ & $.198^{*}$ & $-.025^{*}$ & $.172^{*}$ & $-.071 \dagger$ & .075 & -.017 & $.142^{*}$ & $-.065^{*}$ \\
\hline${ }^{*} p<$ & $<.01$ by $t$ test. $\dagger p<.05$ by $t$ test. & & & & & &
\end{tabular}


Table 3

Average $R^{2}$ Increase from Equation 1 for Standard Sequence Data

\begin{tabular}{llllllllll}
\hline & $I_{n}$ & $R_{n-1}$ & $I_{n-1}$ & $R_{n-2}$ & $I_{n-2}$ & $R_{n-3}$ & $I_{n-3}$ & $R_{n-4}$ & $I_{n-4}$ \\
\hline$S$ & .588 & .052 & .013 & .004 & .028 & .002 & .002 & .002 & .007 \\
$L$ & .636 & .037 & .013 & .001 & .025 & .002 & .002 & .000 & .009 \\
\hline
\end{tabular}

ble 2) and occasionally in the second experiment, this intermodality contrast effect should perhaps be taken seriously. An explanation will be offered in the Discussion section.

Row $L$ of Table 2 shows the same pattern of regression coefficients as does Row $S$, including the reliable intermodality contrast effect of $I_{n-1}$. Row $L$ of Table 3 also closely resembles Row $S$ of that table. The most important previous response was $R_{n-1}$, whereas the most important previous stimulus was $I_{n-2}$. However, previous responses up to a lag of 4 had reliable effects, as did previous stimuli. Thus, these effects should be addressed by psychophysical theory.

Tables 4 and 5 display the results of a similar analysis for the alternating-by-pairs data of Experiment 2. In examining these tables, care must be taken to interpret the various regression coefficients and stimulus and response designations appropriately for the type of sequence involved. For these data, responses to each of $S_{1}, S_{2}, L_{1}$, and $L_{2}$ were analyzed separately, where these symbols have the meanings portrayed in the following sequence:

$$
\ldots S_{1} S_{2} L_{1} L_{2} S_{1} S_{2} L_{1} L_{2} \ldots
$$

Thus, for Row $S_{1}$ in Tables 4 and $5, I_{n}$ is that sound stimulus, $R_{n-1}$ is a response to a light stimulus $\left(L_{2}\right), I_{n-1}$ is that light stimulus $\left(L_{2}\right), R_{n-2}$ is a response to a light stimulus $\left(L_{1}\right), I_{n-2}$ is that light stimulus, and so forth.

Look at Row $S_{1}$ in Table 4. The pattern of regression coefficients in this row is typical of the table. Again, the usual power function exponent, $\gamma$, is reliably different from zero, and of about the same magnitude as in Experiment 1 , considerably smaller than the usual exponent, but only a bit smaller than those obtained for $S_{1}$ with alternating-by-pairs sequences in magnitude $(0.415)$ and category (0.381) matching studies (Ward, 1985). Again, $I_{n}$ adds most to $R^{2}$, here about the same amount as that obtained in other alternating-by-pairs studies. Again, the current response is assimilated to previous responses, up to a lag of 4 , regardless of the modality of their stimuli, and again $R_{n-1}$ adds most to $R^{2}$. Finally, large intramodality contrast effects are found $\left(\alpha_{3}\right.$ and $\left.\alpha_{4}\right)$ up to a lag of 4 , accompanied by much weaker intermodality contrast effects $\left(\alpha_{1}\right.$ and $\left.\alpha_{2}\right)$. This pattern of sequential de- pendencies is repeated for the other rows in Tables 4 and 5 . That the intermodality stimulus contrast effect is much weaker is indicated both by the overall smaller magnitude of the regression coefficients and $R^{2}$ increases, and by the fact that only 4 of the 8 intermodality stimulus coefficients are reliably different from zero. Intermodality response-response assimilation effects are also usually weaker than intramodality effects (e.g., for $\beta_{1}, t=1.998$, $p<.10$ for sound and $t=3.411, p<.01$ for light), as found by Ward (1985) for both magnitude and category matching, but both coefficients are usually large and are almost always reliably different from zero and also yield roughly the same amount of $R^{2}$ increase. The intermodality stimulus-response contrast effects seem different in kind from the intramodality effects, whereas the two types of response-response assimilation effects seem much more similar.

In summary, results of the multiple regression analyses for both experiments are consistent with those of previous mixed-modality scaling studies in that reliable response-response assimilation was found both within and across modalities up to a lag of 4 , and reliable intramodality stimulus-response contrast was also found up to a lag of 4. A much weaker, inconsistent, but occasionally reliable intermodality stimulus-response contrast effect was found for the first time in the present data. This effect seems to be of different origin from the intramodality contrast effect. A possible explanation will be introduced later, in which all of the above-described effects will be characterized within a single framework.

Second-order dependencies. In both ordinary magnitude estimation and category judgment data, as well as in magnitude- and category-matching data from both standard and alternating-by-pairs sequences, the relation between $R_{n}$ and $R_{n-k}$ depends on the separation between $I_{n}$ and $I_{n-k}$ (or between their nominal designations). Typically, the correlation between $\log R_{n}$ and $\log R_{n-k}$ is largest when $I_{n}-I_{n-k}$ is small, and decreases as $I_{n}-I_{n-k}$ increases. The same pattern has been found for crossmodality matching data (e.g., Baird et al., 1980; Ward, 1979), except that the maximum correlation between $\log R_{n}$ and $\log R_{n-k}$ is typically smaller than for other methods. The top part of Figure 2 and all of Figure 3 display the results of analyses of the present data for these second-order dependencies. In the upper part of Figure 2, the results are displayed only for lags of 1 and 2; lags of 3 and 4 give highly similar results and are not displayed. In Figure 3, only results for lags of 1 and 3 are displayed; lags of 2 and 4 give highly similar results and are not displayed. Note that $I_{n}-I_{n-k}$ is given in nominal steps (labeled $S_{n}-S_{n-k}$ ); the differences between adjacent

Table 4

Average Regression Coefficients from Equation 1 for Alternating-by-Pairs Data

\begin{tabular}{cccccccccc}
\hline & $\gamma$ & $\beta_{1}$ & $\alpha_{1}$ & $\beta_{2}$ & $\alpha_{2}$ & $\beta_{3}$ & $\alpha_{3}$ & $\beta_{4}$ & $\alpha_{4}$ \\
\hline$S_{1}$ & $.351^{*}$ & $.181^{*}$ & $-.027 \dagger$ & $.122^{*}$ & $-.021^{*}$ & $.096^{*}$ & $-.074^{*}$ & $.094^{*}$ & $-.060^{*}$ \\
$S_{2}$ & $.336^{*}$ & $.302^{*}$ & $-.111^{*}$ & $.107 \dagger$ & -.024 & .050 & -.006 & $.079 \dagger$ & $-.049^{*}$ \\
$L_{1}$ & $.282^{*}$ & $.077 \dagger$ & .004 & $.092^{*}$ & $-.025 \dagger$ & $.152^{*}$ & $-.068^{*}$ & $.165^{*}$ & $-.072^{*}$ \\
$L_{2}$ & $.288^{*}$ & $.255^{*}$ & $-.052^{*}$ & $.087^{*}$ & $-.023^{*}$ & $.05{ }^{*}$ & -.016 & $.069 \dagger$ & $-.044^{*}$ \\
\hline
\end{tabular}

${ }^{*_{p}}<.01$ by $t$ test. $t_{p}<.05$ by $t$ test. 
Table 5

Average $R^{2}$ Increase from Equation 1 for Alternating-by-Pairs Data

\begin{tabular}{llllllllll}
\hline & $I_{n}$ & $R_{n-1}$ & $I_{n-1}$ & $R_{n-2}$ & $I_{n-2}$ & $R_{n-3}$ & $I_{n-3}$ & $R_{n-4}$ & $I_{n-4}$ \\
\hline$S_{1}$ & .752 & .017 & .006 & .005 & .002 & .003 & .012 & .002 & .005 \\
$S_{2}$ & .717 & .015 & .034 & .002 & .003 & .002 & .001 & .002 & .005 \\
$L_{1}$ & .713 & .013 & .003 & .003 & .002 & .003 & .017 & .002 & .015 \\
$L_{2}$ & .749 & .018 & .018 & .002 & .002 & .001 & .001 & .002 & .004 \\
\hline
\end{tabular}

stimulus intensities were equal in log units. The correlations plotted are the averages of individual subjects' correlations; each such correlation was calculated on at least 15 pairs of values, and each average correlation represents at least five individual subjects' correlations.

For the data of the standard sequence (Experiment 1), the correlation between $\log R_{n}$ and $\log R_{n-k}$ depends on $S_{n}-S_{n-k}$ in the same way as for other scaling data, forming an inverted $\mathrm{V}$ pattern. The corresponding correlations for the Experiment 2 data are a little more variable, since they are based on fewer pairs of values, but they display the same basic pattern. Both first- and second-order assimilative response-response dependencies are the same regardless of which modality is being responded to. Furthermore, at least in the present studies, the responseresponse correlations for small separations of $S_{n}$ and $S_{n-k}$ can be quite large, approaching the values typically observed in magnitude estimation and category judgment.

Variability of response ratios. Previous studies of magnitude estimation and category judgment have found that the coefficient of variation for ratios of successive responses (standard deviation/mean, or $\mathrm{s} / \mathrm{m}$ ) varies as a function of $I_{n}-I_{n-1}$ (e.g., Green \& Luce, 1974; Ward, 1985). However, this result does not seem to be true for cross-modality matches of area to loudness or vice versa (Baird et al., 1980). The present data were analyzed for this effect, and the bottom part of Figure 2 and all of Figure 4 display the results of these analyses for the data of Experiment 1 and Experiment 2, respectively. The results for lags of 1 and 2 are displayed for Experiment 1; results for lags of 3 and 4 were highly similar and are not displayed. Similarly, results for lags of 1 and 3 are displayed for Experiment 2; results for lags of 2 and 4 were highly similar and are not displayed.

Consider first the intramodality effects (e.g., $S$ on $S$ or $L$ on $L$ ) displayed in Figure 2. Not unexpectedly, since a lag of 2 is involved, there is scant evidence for the usual $\mathrm{V}$-shaped pattern. The result for a lag of 1 , an intermodality effect, is the same. Figure 4 gives a similar set of results, even for intramodality lags of 1 , for which lag Ward's (1985) magnitude- and category-matching data showed the V-shaped pattern. Consistent with other crossmodality matching studies, there is little evidence here for a dependence of $s / m$ on $I_{n}-I_{n-k}$. Since this pattern has been taken as evidence of the operation of an attention band (see Green, Luce, \& Duncan, 1977) in such judgments, it may be either that the attention band does not operate for cross-modality matches or that the larger variability of cross-modality matching responses obscures its effect.

\section{DISCUSSION}

The results of the two experiments described above allow several general conclusions. First, cross-modality matching of an "easy" sensory continuum to two "difficult" continua gives derived cross-modality matching functions similar to those obtained using the methods of magnitude or category matching, at least for duration, light, and sound. Thus, when verbal or numerical responses are difficult to obtain or undesirable, double cross-modality matching can be used to obtain reliable derived matching functions. To extend the generality of this conclusion, more "easy" continua should be tried and other types of "difficult" continua should be used, especially those involving the minor senses. However, when it is possible to use duration, it does provide an especially useful "easy" response continuum.

Second, there is a great deal of similarity between the various sequential dependencies observed with the present method and those observed in magnitude and category matching, and in magnitude estimation, category judgment, and cross-modality matching in non-mixed-modality situations. Assimilation of the current response to previous responses is observed reliably up to a lag of 4 in mixed-modality situations, although the only previous response accounting for an appreciable $R^{2}$ increase is $R_{n-1}$. Moreover, this response-response assimilation has the same form both within and across stimulus modalities, although regression coefficients for intermodality assimilation are somewhat smaller. Also, as in magnitude and category matching, current cross-modality matching responses are contrasted with stimuli of the same modality as the current stimulus up to a lag of 4 . These effects are of appreciable magnitude at least up to a lag of 3 , and are highly reliable even at a lag of 4 , although they seldom add more than 0.01 to $R^{2}$ at that lag.

In contrast to magnitude and category matching, however, the present study occasionally found reliable intermodality contrast effects at lags of 1 or 2 , although the regression coefficient estimates for these stimuli were much smaller than those for intramodality effects. Indeed, they were of the same magnitude as effects in earlier studies that were not reliably different from zero. If these effects are real, they are surely much weaker than the intramodality contrast effects found in all mixedmodality situations, and may be reliable enough to demand attention only in double cross-modality matching situations.

Another interesting and potentially important difference between cross-modality matching and verbal or numerical judgments is the relative absence of the dependence of $s / m$ for $R_{n} / R_{n-1}$ on $I_{n}-I_{n-1}$. This effect also failed to appear strongly in the present data, although it is present for magnitude and category matching (Ward, 1985). It 

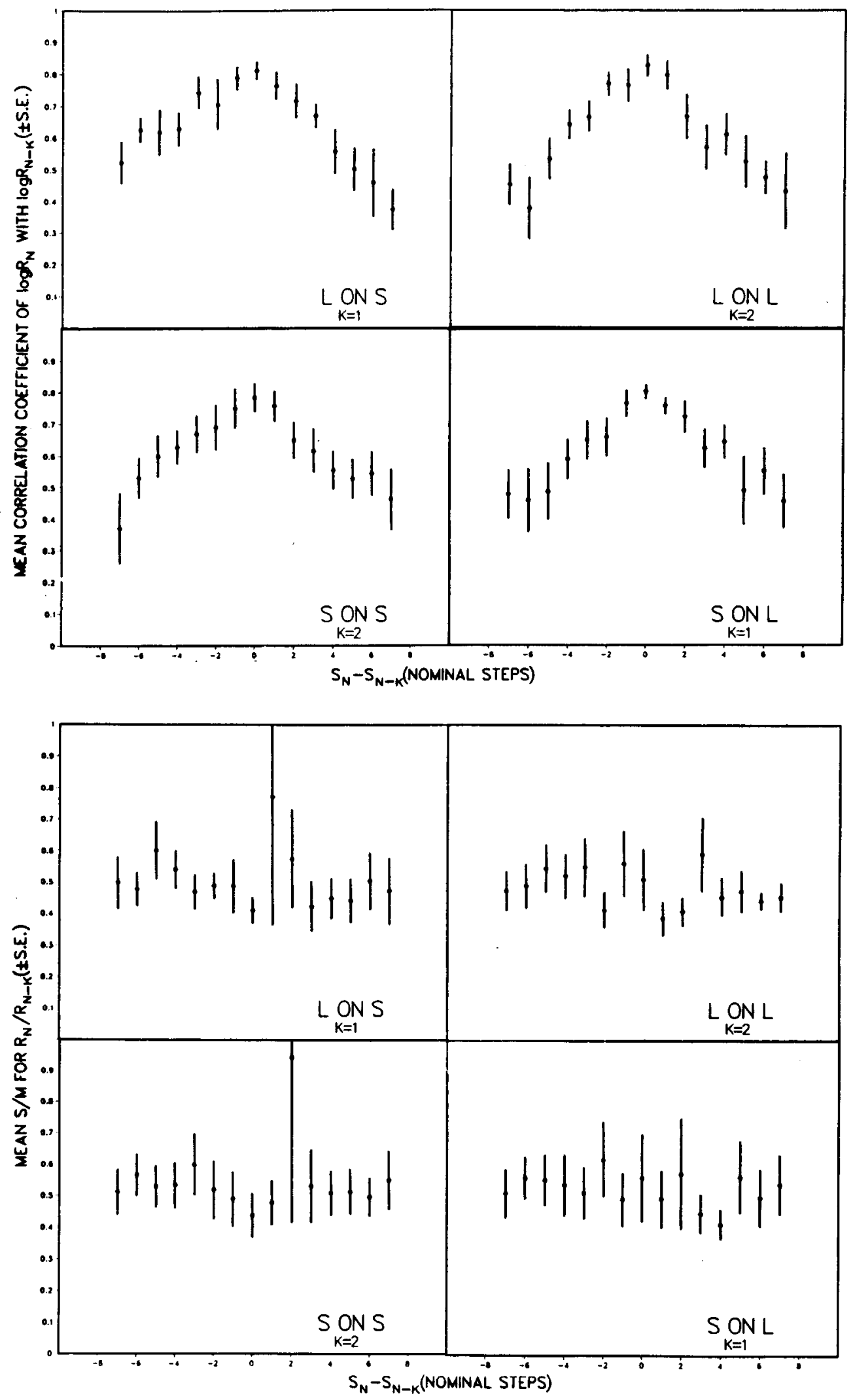

Figure 2. Top: Plots of the correlation between $\log R_{n}$ and $\log R_{n-k}$ as a function of $S_{n}-S_{n-k}$ for lags of $1(k=1)$ and $2(k=2)$ for the standard sequence data. Bottom: Plots of $s / m$ for $R_{n} / R_{n-k}$ as a function of $S_{n}-S_{n-k}$ for lags of $1(k=1)$ and $2(k=2)$ for the standard sequence data. 

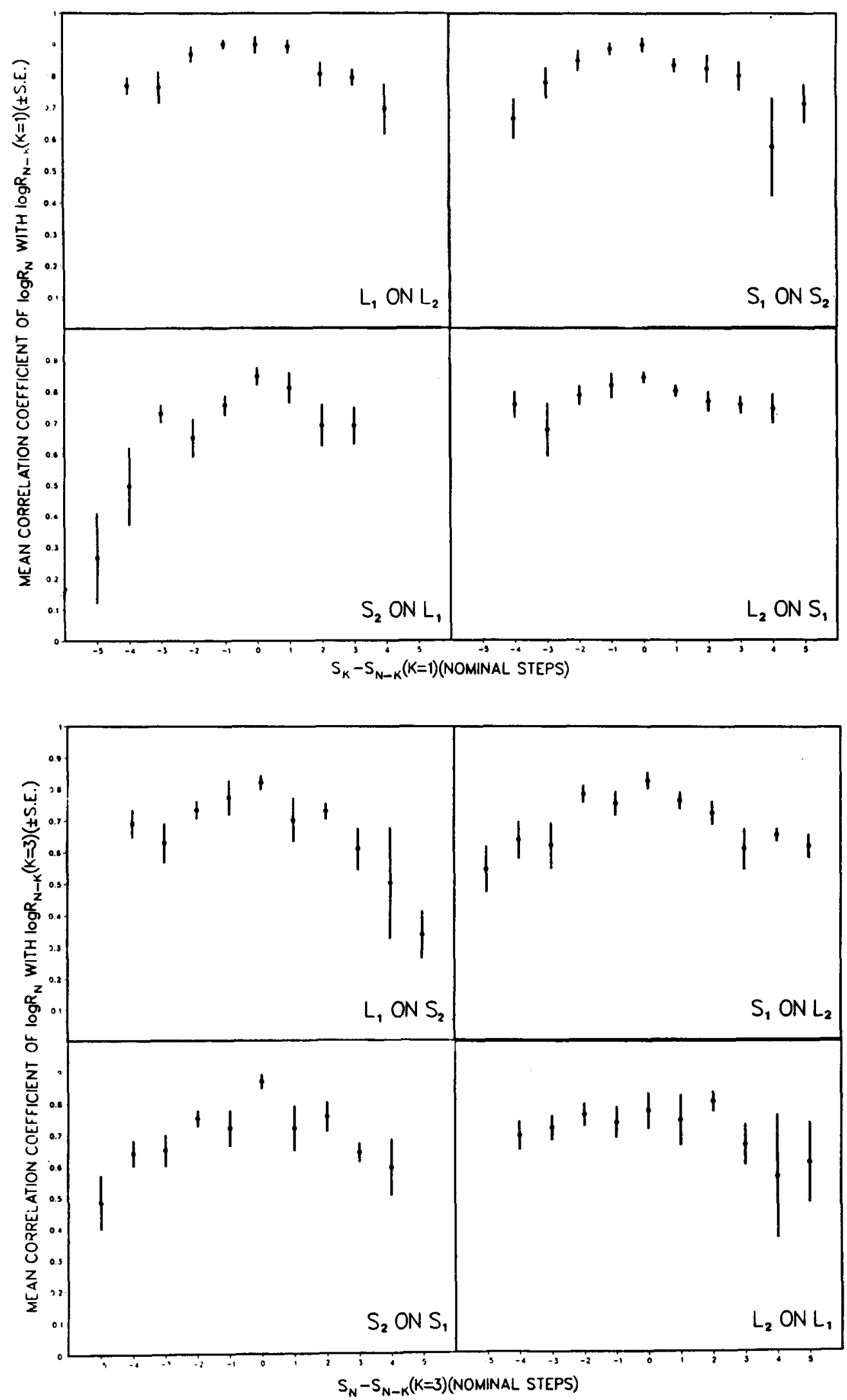

Figure 3. Plots of the correlation between $\log R_{n}$ and $\log R_{n-k}$ as a function of $S_{n}-S_{n-k}$ for lags of $1(k=1)$ and $3(k=3)$ for the alternating-by-pairs sequence data. 

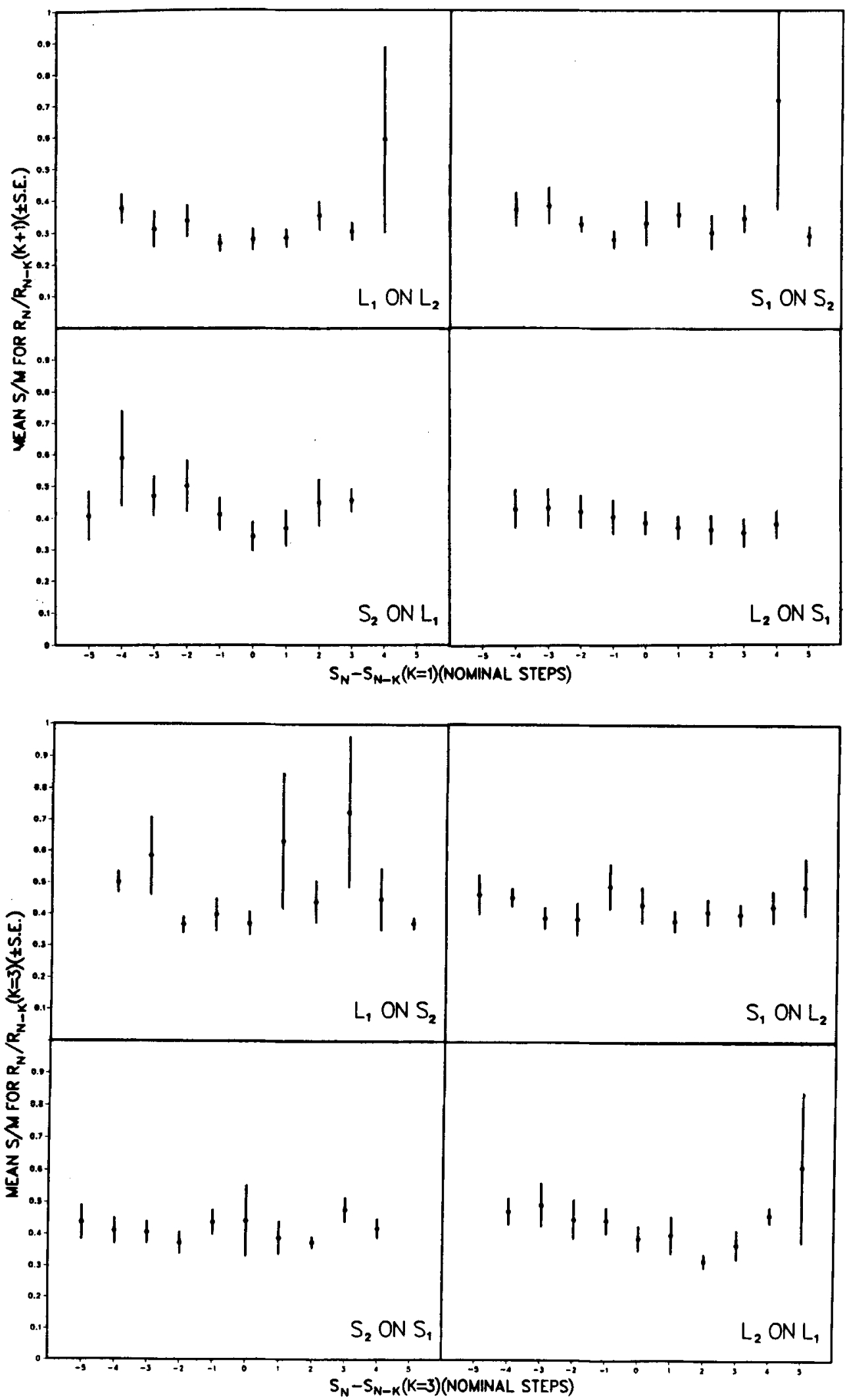

Figure 4. Plots of $s / m$ for $R_{n} / R_{n-k}$ as a function of $S_{n}-S_{n-k}$ for lags of $1(k=1)$ and $3(K=3)$ for the alternating-by-pairs sequence data. 
is difficult to say what this means, but one possibility is that having to attend to a different sensory modality in order to produce the response in cross-modality matching interferes with the ability to locate an attention band precisely enough at the location of the previous samemodality stimulus (cf. Baird et al., 1980). This idea is consistent with the observation that in mixed-modality scaling, the effect appears only for intramodality comparisons and only for a lag of 1 (Ward, 1982, 1985). Apparently, intervening stimuli and/or responses interfere with the effect for greater lags.

It may be useful to conceptualize all of the sequential dependencies discovered in mixed-modality and other scaling situations within a more general framework. This framework assumes that the various effects arise from different mechanisms that operate within different levels of stimulus processing, much as did Ward's (1979) theory of fuzzy judgment. At least three general levels are identified: sensory, perceptual, and cognitive. The levels are characterized functionally. It is assumed that the primary function of the sensory level is to produce an internal representation of the stimulus that is sharply differentiated from other temporally and spatially contiguous stimuli. There are several sensory mechanisms that could contribute to this function, but the most plausible is lateral inhibition. In fact, nearly all of the low-level sensory mechanisms that involve lateral inhibition show contrast between stimuli presented either simultaneously or successively (see, e.g., Békésy, 1967; Cornsweet, 1970; Levine \& Grossberg, 1976).

The primary function of the perceptual level is to partition the sharply differentiated internal stimulus representations into ecologically valid functional categories. Mechanisms at this level attempt to produce an interpretation of the stimulus situation that is as simple as possible (cf. Hatfield \& Epstein, 1985) and that is consistent with the current model of the task environment based on previous and expected future categorizations (cf. Wickens, Kramer, Vanasse, \& Donchin, 1983). These requirements create a passive expectancy of category repetition (cf. Kahneman \& Tversky, 1982), which may result in assimilation, or pooling, of successive categorizations (cf. Coren \& Girgus, 1978).

Finally, the cognitive level deals with abstractions. It searches for patterns among categorizations so that more abstract models can be constructed to aid in dealing with the environment. This type of processing in principle could introduce either contrastive or assimilative tendencies, but would be expected to be contrastive when dealing with sequences. This is because people apparently do not understand randomness; a truly random sequence has too many runs, which typically are interpreted as meaningful patterns, to be seen as lacking patterns. This results in the well-known gambler's fallacy, in which the estimated likelihood of an alternation in a nominally random sequence increases with the length of a run. This would result in an active expectancy of change when viewing a sequence of stimuli, and a tendency to resolve response uncertainty in the direction of contrast with previous stimuli.

When applied to a sequence of mixed-modality scaling judgments, this framework may clarify the possible sources of the various sequential dependencies observed. First, the assimilative response-response dependencies seem to originate at the perceptual level. Whether the effects are inter- or intramodality is immaterial, since the categorization required is at the somewhat abstract level of "sensory magnitude," and also the perceptual level commonly integrates information across different sensory systems in order to arrive at a coherent interpretation of the world. Second, the intramodality response-stimulus contrast effects seem to be of sensory origin, since they are not accompanied by intermodality effects of the same magnitude, as would be expected if they were of cognitive origin. Finally, the weak, inconsistent intermodality response-stimulus contrast effects may be of cognitive origin; perceptual origin is ruled out by the nature of the effects (contrastive), and sensory origin is ruled out by the fact that low-level sensory internal representations most probably do not interact across modalities, since they are localized in quite different parts of the sensory cortex. The fact that effects only appear with enough consistency to be noticeable when responses are made via cross-modality matching is probably a consequence of the larger amount of response uncertainty there.

\section{REFERENCES}

BaIrD, J. C., Green, D. M., \& Luce, R. D. (1980). Variability and sequential effects in cross-modality matching of area and loudness. Joumal of Experimental Psychology: Human Perception \& Performance, 6, 277-289.

BÉkÉsY, G. voN (1967). Sensory inhibition. Princeton, NJ: Princeton University Press.

Coren, S., \& Girgus, J. S. (1978). Seeing is deceiving: The psychology of visual illusions. Hillsdale, NJ: Erlbaum.

CoRnsweet, T. N. (1970). Visual perception. New York: Academic Press.

DANING, R. (1983). Intraindividual consistencies in cross-modal matching across several continua. Perception \& Psychophysics, 33, 516-522.

Green, D. M., \& LUCE, R. D. (1974). Variability of magnitude estimates: A timing theory analysis. Perception \& Psychophysics, 15, 291-300.

Green, D. M., Luce, R. D., \& Duncan, J. E. (1977). Variability and sequential effects in magnitude production and estimation of auditory intensity. Perception \& Psychophysics, 22, 450-456.

Hatfield, G., \& EPstein, W. (1985). The status of the minimum principle in the theoretical analysis of visual perception. Psychological Bulletin, 97, 155-186.

Jesteadt, W., Luce, R. D., \& Green, D. M. (1977). Sequential effects in judgments of loudness. Journal of Experimental Psychology: Human Perception \& Performance, 3, 92-104.

Kahneman, D., \& Tversky, A. (1982). Variants of uncertainty. Cognition, 11, 143-157

KRANTZ, D. (1972). A theory of magnitude estimation and crossmodality matching. Journal of Mathematical Psychology, 9, 168-199.

LEVINE, D. S. , \& GrossberG, S. (1976). Visual illusions in neural networks: Line neutralization, tilt aftereffect, and angle expansions. Journal of Theoretical Biology, 61, 477-504.

Marks, L. E. (1974). On scales of sensation: Prolegomena to any future psychophysics that will be able to come forth as science. Perception \& Psychophysics, 16, 358-376. 
Stevens, J. C., \& Marks, L. E. (1980). Cross-modality matching functions generated by magnitude estimation. Perception \& Psychophysics, 27, 379-389.

Stevens, S. S. (1975). Psychophysics. New York: Wiley.

Teghtsoonian, M., \& Teghtsoonian, R. (1983). Consistency of individual exponents in cross-modal matching. Perception \& Psychophysics, 33, 203-214.

WARD, L. M. (1972). Category judgments of loudnesses in the absence of an experimenter-induced identification function: Sequential effects and power function fit. Journal of Experimental Psychology, 94, 179-184.

WARD, L. M. (1975). Sequential dependencies and response range in cross-modality matches of duration to loudness. Perception \& Psychophysics, 18, 217-223.

WARD, L. M. (1979). Stimulus information and sequential dependencies in magnitude estimation and cross-modality matching. Journal of Experimental Psychology: Human Perception \& Performance, 5, 444-459.

WARD, L. M. (1982). Mixed-modality psychophysical scaling: Sequential dependencies and other properties. Perception \& Psychophysics, 31, 53-62.

WARD, L. M. (1985). Mixed-modality psychophysical scaling: Interand intramodality sequential dependencies as a function of lag. Perception \& Psychophysics, 38, 512-522.

Wickens, C., Kramer, A., Vanasse, L., \& Donchin, E. (1983). Performance of concurrent tasks: A psychophysiological analysis of reciprocity of information-processing resources. Science, 221, 1080-1082.

(Manuscript received January 31, 1986; revision accepted for publication April 15, 1986.) 